\title{
Oxaliplatin-based hyperthermic intraperitoneal chemotherapy with single drug versus multiple drug treatment for colorectal cancer with peritoneal metastases: an observational cohort study
}

\author{
Petter Frühling, Lana Ghanipour, Paul Dranichnikov, Malin Enblad, Helgi Birgisson, Peter H. Cashin \\ Department of Surgical Sciences, Section of Surgery, Uppsala University, Akademiska Sjukhuset, Uppsala, Sweden \\ Contributions: (I) Conception and design: All authors; (II) Administrative support: All authors; (III) Provision of study materials or patients: All \\ authors; (IV) Collection and assembly of data: All authors; (V) Data analysis and interpretation: All authors; (VI) Manuscript writing: All authors; (VII) \\ Final approval of manuscript: All authors. \\ Correspondence to: Dr. Petter Frühling, MD. Department of Surgical Sciences, Section of Surgery, Uppsala University, Akademiska Sjukhuset, 75185 \\ Uppsala, Sweden. Email: Petter.fruhling@surgsci.uu.se.
}

\begin{abstract}
Background: Long-term survival for selected patients with peritoneal metastases (PM) from colorectal cancer (CRC) is possible when treated with cytoreductive surgery (CRS) and hyperthermic intraperitoneal chemotherapy (HIPEC). The objective of this study was to compare three different oxaliplatin-based (OX)HIPEC regimens. Primary end-point was disease-free survival (DFS), and secondary endpoints, morbidity and overall survival (OS).
\end{abstract}

Methods: This is a retrospective study of all patients with colorectal PM treated with CRS and HIPEC between 2004 and 2015 from the prospectively maintained Uppsala HIPEC database. One hundred and thirty-three patients were identified. Three HIPEC regimens were included: OX-HIPEC, OX-HIPEC + post-operative intraperitoneal chemotherapy (EPIC) with 5-fluorouracil (5-FU), and oxaliplatin-irinotecanbased (OXIRI)-HIPEC. Multivariable Cox regression for DFS was performed.

Results: Sixty-one patients received OX-HIPEC, 24 patients received OX-HIPEC + 5-FU EPIC, and 48 patients received OXIRI-HIPEC. The DFS for the OX-HIPEC group was 10.5 months, OX-HIPEC + EPIC 11.9 months, and OXIRI-HIPEC 13.4 months (OX-HIPEC vs. OXIRI HIPEC, P=0.049). The morbidity and OS did not differ between the groups. In the multivariable analysis, low peritoneal cancer index (PCI), absence of liver metastases, low completeness of cytoreduction (CC) score, and multiple drug (EPIC or OXIRI) HIPEC regimen were independent prognostic factors for DFS.

Conclusions: This study showed improved DFS with an intensification of HIPEC by adding irinotecan or EPIC compared to oxaliplatin alone without an increase in morbidity or mortality.

Keywords: Colorectal cancer (CRC); peritoneal metastases (PM); cytoreductive surgery (CRS); hyperthermic intraperitoneal chemotherapy (HIPEC); oxaliplatin

Submitted Nov 06, 2020. Accepted for publication Jan 18, 2021.

doi: 10.21037/jgo-20-494

View this article at: http://dx.doi.org/10.21037/jgo-20-494

\section{Introduction}

For a long period of time colorectal cancer (CRC) with peritoneal metastases $(\mathrm{PM})$ was considered a palliative situation with a median survival of 5-7 months (1). In the past, these patients received palliative chemotherapy. Cytoreductive surgery (CRS) is a relatively new development in the surgical treatment of advanced gastrointestinal cancer. In brief, the general consensus is that CRS should only be used in patients without hematogenic, extra-abdominal or retro-peritoneal lymph node metastases, with the exception of 1-3 liver metastases (2). The median survival for patients in whom complete 
cytoreduction is achieved is around 30 months (3-5). A recent comprehensive meta-analysis by Huang et al. (6), conclude that CRS and hyperthermic intraperitoneal chemotherapy (HIPEC) improve median OS in patients with CRC with PM.

The HIPEC method is heterogenous, and the treatment lacks standardization. The recent results from the PRODIGE-7 study, published as an abstract only, suggest that the addition of oxaliplatin-based (OX)-HIPEC does not influence overall survival (OS) (7). Consequently, it would be of interest to evaluate if an intensification of HIPEC by adding EPIC or irinotecan to OX-HIPEC could influence the outcome. The aim of the present study was to examine the impact of three different HIPEC regimens on diseasefree survival (DFS), OS, and morbidity and mortality. The three different regimens that are studied are OX-HIPEC, OX-HIPEC + post-operative intraperitoneal chemotherapy (EPIC) with 5-fluorouracil (5-FU), and oxaliplatinirinotecan-based (OXIRI)-HIPEC + EPIC with 5-FU, and OXIRI-HIPEC. We present the study in accordance with Strengthening the Reporting of Observational Studies in Epidemiology (STROBE) reporting checklist (8) (available at http://dx.doi.org/10.21037/jgo-20-494).

\section{Methods}

The study was conducted in accordance with the Declaration of Helsinki (as revised in 2013), and was approved by the regional Ethical Review Board, DnR $2013 / 203$. Given the retrospective design of the study, the Ethical Board did not consider a written informed consent to be necessary.

\section{Study design}

This study used data from a prospectively maintained HIPEC database at Uppsala University Hospital. All patients with colorectal PM who underwent CRS and OXHIPEC \pm EPIC or OXIRI-HIPEC from 1st January 2004 to 31st December 2015, were included (Figure 1). Prior to treatment, all patients were discussed in a multi-disciplinary meeting to ensure that they were eligible for CRS and HIPEC. The eligibility criteria were a histologically confirmed diagnosis of CRC and PM, no distant metastasis, WHO performance $\leq 2$, and normal renal, liver and hematopoietic functions. The presence of one to three liver metastases was not considered a contraindication, as long as they were easily resectable simultaneously as CRS.
Clinicopathological data were obtained from patient records and the Uppsala University Hospital HIPEC database. Variables that were collected included gender, age, prior surgical score (PSS), peritoneal cancer index (PCI), completeness of cytoreduction (CC) score, and type of HIPEC treatment (OX-HIPEC, OX-HIPEC + EPIC, and OXIRI-HIPEC), location of primary tumor (colon or rectum), and node positive primary. Furthermore, in hospital, and 90-day mortality and morbidity, as well as the use of adjuvant chemotherapy, and histopathology were collected from the patient records.

\section{Surgical procedure}

At laparotomy the resection of PM followed the principles of Sugarbaker (9). The PCI was calculated, which ranges from 1 to 39. This is a semi-quantitative measure of the tumor burden, obtained by calculating the lesion size scores [0-3] in the 13 regions of the abdomen (10). The CC score was calculated (11), where CC-0 corresponds to no visible tumor tissue left in the abdomen. CC-1 indicates residual tumor nodules $<2.5 \mathrm{~mm}$ in diameter, and CC-2 means there are remaining nodules between 2.5 and $25 \mathrm{~mm}$, and CC- 3 means remaining nodules $>25 \mathrm{~mm}$. If the PM was considered too extensive, so as to preclude a complete macroscopic resection the procedure was aborted and CRS not performed. These patients were not included in this study.

\section{Chemotherapy agents}

At the start of the Uppsala HIPEC program, OX-HIPEC was given together with 5 -FU EPIC for 5 days. After a short period, the EPIC addition was discontinued due to preliminary reports of increased morbidity, and instead combination OXIRI-HIPEC was used. This continued until a national program in Sweden was put in place around 2011, in which case all Swedish centers used single OX-HIPEC as the common standard (Figure 2). The HIPEC treatment was given according to the coliseum method (12), where either oxaliplatin $\left(460 \mathrm{mg} / \mathrm{m}^{2}\right)$ was administered intraperitoneally for 30 minutes or in combination with irinotecan $\left(400 \mathrm{mg} / \mathrm{m}^{2}\right.$ for both drugs) for 30 minutes. All three different HIPEC regimens in this study included a single bolus-dose of IV 5-FU at $400 \mathrm{mg} / \mathrm{m}^{2}$ intraoperatively (sometimes dose reduced if necessary). The intra-abdominal temperature was targeted at $41-43{ }^{\circ} \mathrm{C}$, and controlled with three thermal probes, 


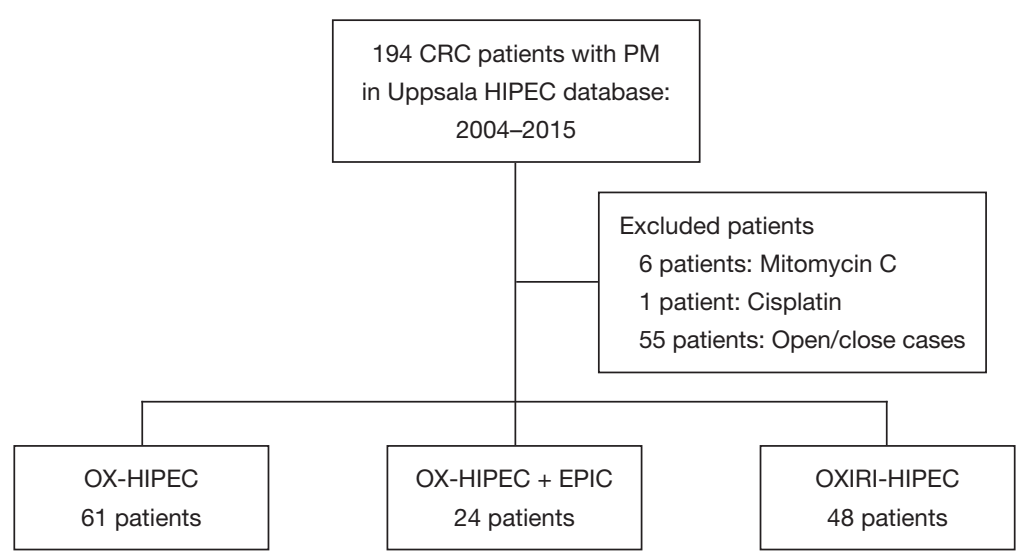

Figure 1 Flowchart of patients with CRC and PM (n=133). CRC, colorectal cancer; PM, peritoneal metastases; HIPEC, hyperthermic intraperitoneal chemotherapy; OX-HIPEC, oxaliplatin-based HIPEC; EPIC, post-operative intraperitoneal chemotherapy; OXIRI-HIPEC, oxaliplatin-irinotecan-based HIPEC.

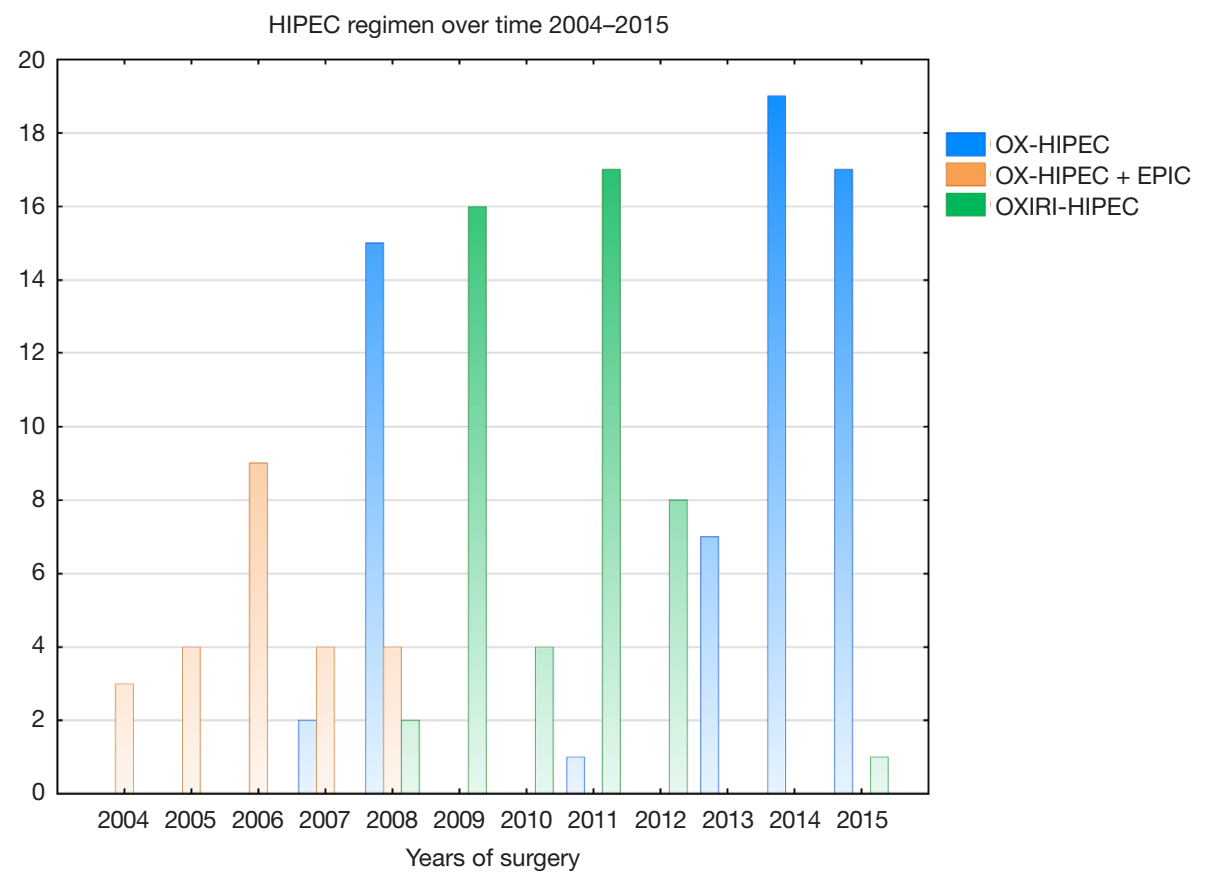

Figure 2 Description of which HIPEC regimen, that was used during the study period 2004-2015. HIPEC, hyperthermic intraperitoneal chemotherapy; OX-HIPEC, oxaliplatin-based HIPEC; EPIC, post-operative intraperitoneal chemotherapy; OXIRI-HIPEC, oxaliplatinirinotecan-based HIPEC.

with a flow-rate of $1-2 \mathrm{~L} / \mathrm{min}$. Electrolyte-free glucose $(50 \mathrm{mg} / \mathrm{mL})$ was used for perfusion. The EPIC treatment was a normothermic 5 -FU treatment administered daily at post-operative day $1-5$ at $500-600 \mathrm{mg} / \mathrm{m}^{2}$ in $250 \mathrm{~mL}$ of saline solution that was injected through an abdominal drainage catheter. The drains were clamped overnight and then opened for a few hours before the next administration.

\section{Follow-up after surgery}

All patients were followed-up 4 weeks post-operatively in 
the outpatient clinic. Thereafter, follow-up was done, as a minimum, every 6 months for 2 years and then yearly, with contrast enhanced computed tomography (CE-CT) of the chest and abdomen. The median follow-up time was defined as median observation time for those patients who were alive at the end of the study. Information regarding recurrence was retrieved from patient medical records, and death and cause of death from the Swedish Data Registry.

\section{Statistical analysis}

DFS was defined as the time interval between date of surgery and documented recurrence or death from any cause. OS was defined as the time period between date of surgery and death from any cause. Time was censored at the last follow-up for patients that were still alive or lost to follow-up. Survival was analyzed with the Kaplan-Meier method, and compared with the log-rank test. Pearson's chi ${ }^{2}$ test was used to compare differences in categorical variables between the three groups, and Kruskal-Wallis test was used when comparing continuous variables. A univariable and multivariable Cox proportional hazard regression model was used to assess the relationship between a predetermined set of clinicopathological variables and DFS. All analyses were carried out with Statistica 13.4.0.14 (TIBCO Software Inc., Palo Alto, CA, USA), and the level of statistical significance was defined as a two-sided $\mathrm{P}<0.05$.

\section{Results}

\section{Patient characteristics}

This study included 133 patients (Table 1). Median age for the whole study population was 59 years. A majority of patients were women, 76 (57\%) compared to 57 (43\%) men. Sixty-one patients were treated with OX-HIPEC, 24 patients with OX-HIPEC + EPIC, and 48 patients with OXIRI-HIPEC. The primary tumor was located in the colon in $92 \%(\mathrm{n}=123)$ of the patients and in the rectum in $8 \%(n=10)$. Liver metastases were present in $25 \%(n=6)$ of the patients in the OX-HIPEC + EPIC compared to $7 \%(n=4)$ and $13 \%(n=6)$ in the OX-HIPEC and OXIRIHIPEC groups, respectively $(\mathrm{P}=0.06)$.

Synchronous colorectal PM were present in $71 \%$ of the cases in the OXIRI-HIPEC group compared to $58 \%$ and $56 \%$, in OX-HIPEC + EPIC and OX-HIPEC groups, respectively. There was a statistical difference between the groups in terms of neoadjuvant chemotherapy $(\mathrm{P}=0.004)$ and node positive primary $(\mathrm{P}=0.003)$, but not in terms of adjuvant chemotherapy $(\mathrm{P}=0.64)$. Overall, the three groups showed similar demographic and patient characteristics (Table 1). The median follow-up time was 86 (IQR, 52-117) months.

\section{Surgical results}

Median PCI was 14 in the whole cohort. A CC-0 resection was achieved in $89 \%(n=118)$ of the patients. There was no difference in CC-scores between the groups $(\mathrm{P}=0.094)$. Resection of liver metastases was performed in $16 \%$ $(\mathrm{n}=35)$ of patients. There was a difference in operating time between the groups $(\mathrm{P}=0.004)$. Mean operating time in the OX-HIPEC was 456 minutes (SD: 197), compared to 542 minutes (SD: 155) in the HIPEC + EPIC, and 522 minutes (SD: 137) in the OXIRI-HIPEC.

\section{Post-operative mortality and morbidity}

In-hospital mortality occurred in 2 cases (1.5\%). Postoperatively, 14 patients were re-operated due to complications (Table 2). There were no differences in morbidity, defined as Clavien-Dindo grade 3 or 4 , between the groups $(\mathrm{P}=0.2)$. The overall rate of morbidity was $26 \%(n=35)$. Post-operative neutropenia occurred in $44 \%(n=28)$ of the patients in the OXIRI-HIPEC group, compared to only $1.6 \%(\mathrm{n}=1)$ of patients in the OX-HIPEC group ( $\mathrm{P} \leq 0.0001)$ (Table 2) (13). A secondary analysis that compared single-drug HIPEC (OX-HIPEC) and multipledrug HIPEC (OX HIPEC + EPIC or OXIRI-HIPEC) revealed no differences in Clavien-Dindo grades 3-4, or return to operation theatre post-operatively (Table 2). The rate of peritoneal recurrence was improved from $49 \%$ to $32 \%$ with a borderline $\mathrm{P}$ value $(\mathrm{P}=0.051)$.

\section{Survival analyses}

In the univariable analysis PCI, and a CC score 1-3 vs. 0, were associated with worse DFS (Table 3). In the multivariable Cox regression model PCI (HR: 1.03, 95\% CI: 1.01-1.06), liver metastases (HR: 2.29, 95\% CI: $1.25-$ 4.19), and CC score 1-3 vs. 0 (HR: 5.19, 95\% CI: $2.46-$ 11.0), remained significantly associated with poor DFS. Moreover, combination drug-therapy with HIPEC + EPIC or OXIRI-HIPEC, was associated with better DFS (HR: 0.48, 95\% CI: 0.25-0.92, and HR: 0.59, 95\% CI: 0.37-0.94, respectively), than monotherapy (OX-HIPEC) (Table 3).

At 24 months 48 (36\%) patients had a systemic recurrence, 
Table 1 Demographics and baseline characteristics of patients with CRC with PM

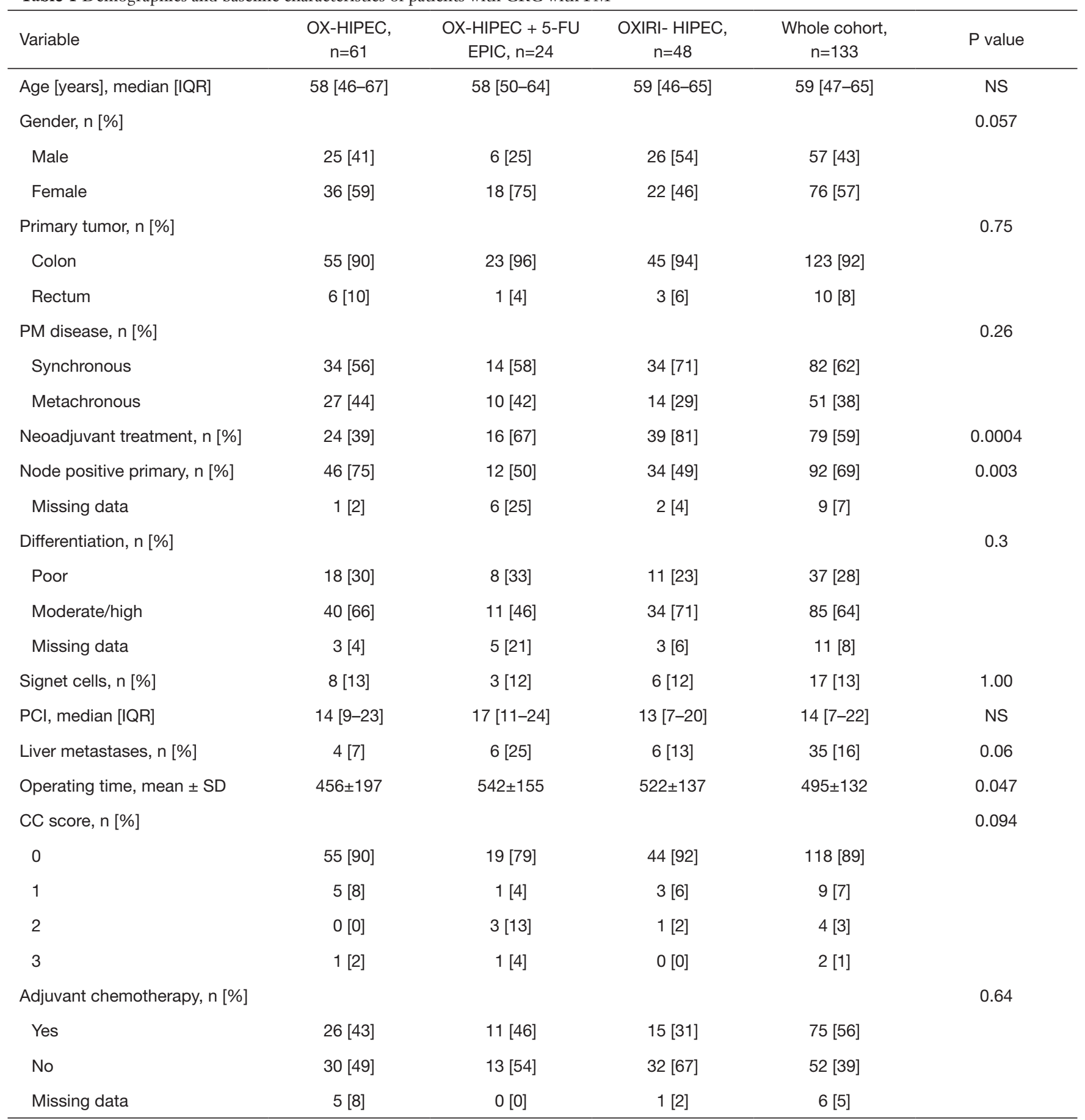

CRC, colorectal cancer; PM, peritoneal metastases; HIPEC, hyperthermic intraperitoneal chemotherapy; OX-HIPEC, oxaliplatin-based HIPEC; 5-FU, 5-fluorouracil; EPIC, post-operative intraperitoneal chemotherapy; OXIRI-HIPEC, oxaliplatin-irinotecan-based HIPEC; PCI, peritoneal cancer index; $\mathrm{CC}$, completeness of cytoreduction. 
Table 2 Morbidity outcomes and recurrence analysis

\begin{tabular}{|c|c|c|c|c|c|}
\hline Variable & OX-HIPEC, $n=61$ & OX-HIPEC + EPIC, n=24 & OXIRI-HIPEC, $\mathrm{n}=48$ & Whole cohort, $n=133$ & $P$ value \\
\hline Return to OR postop, n [\%] & $6[10]$ & $4[17]$ & $4[8]$ & $14[11]^{*}$ & 0.4 \\
\hline Clavien-Dindo grade 3-4, n [\%] & 19 [31] & 7 [29] & 9 [19] & 35 [26] & 0.2 \\
\hline 3 & $15[25]$ & 7 [29] & $7[15]$ & 29 [22] & \\
\hline In-hospital mortality, n [\%] & $1[2]$ & $1[4]$ & $0[0]$ & 2 [2] & \\
\hline Any grade neutropenia, $\mathrm{n}[\%]$ & $1[2]$ & $6[25]$ & $21[44]$ & $28[21]$ & $<0.0001$ \\
\hline \multicolumn{6}{|l|}{ Recurrences at 24 months } \\
\hline Systemic recurrence & $18[30]$ & 9 [37] & $21[44]$ & $48[36]$ & 0.2 \\
\hline Missing data & $5[8]$ & $1[4]$ & $3[6]$ & $9[7]$ & \\
\hline $\begin{array}{l}\text { Secondary analysis: single drug } \\
\text { HIPEC vs. multiple drug HIPEC }\end{array}$ & $\begin{array}{l}\text { Single drug HIPEC, } \\
\qquad \mathrm{n}=61\end{array}$ & $\begin{array}{l}\text { Multiple drug } \\
\text { (OX EPIC and O }\end{array}$ & $\begin{array}{l}9 \text { HIPEC } \\
\text { XIRI), } n=72\end{array}$ & & \\
\hline Return to OR postop, n [\%] & $6[10]$ & $8[11]$ & & $14[11]^{\star}$ & 0.9 \\
\hline Clavien-Dindo grade 3-4, n [\%] & 19 [31] & $16[22$ & & 35 [26] & 0.3 \\
\hline Peritoneal recurrences, $\mathrm{n}$ [\%] & $30[49]$ & $23[32$ & & $53[40]$ & 0.051 \\
\hline
\end{tabular}

*, Reasons for re-operation ( $n=14)$ : 2 bleeding, 2 anastomotic leakage, 2 sepsis unclear abdominal cause, 3 small bowel or gastric perforations, 2 wound dehiscence, 1 mesh-caused bowel obstruction, 1 unclear severe postop pain, 1 ureter perforation. HIPEC, hyperthermic intraperitoneal chemotherapy; OX-HIPEC, oxaliplatin-based HIPEC; EPIC, post-operative intraperitoneal chemotherapy; OXIRI-HIPEC, oxaliplatin-irinotecan-based HIPEC; OR, operating room.

and $53(40 \%)$ patients had a peritoneal recurrence. There were no differences in systemic recurrence between the groups; however, peritoneal recurrences showed a trend toward improved recurrence rates, $\mathrm{P}=0.08$ (Table 2). Figure 3 shows DFS for the respective groups. In the OX-HIPEC group DFS was 10.5 months compared to 13.4 months in OXIRI-HIPEC $(\mathrm{P}=0.049)$. DFS was 11.9 months in the OXHIPEC + EPIC group. In a subgroup analysis excluding CC 1-3 and liver metastases, DFS was 11.7 months in OXHIPEC, OX-HIPEC + EPIC 23.6 months, and OXIRIHIPEC 18.6 months (Figure 4).

Overall median survival in the OX-HIPEC group was 31.2 months, and in the OX-HIPEC + EPIC, and OXIRIHIPEC groups 24 and 36.5 months, respectively (Figure 5). There were no statistical differences between the groups.

\section{Discussion}

Intensification of OX-HIPEC with either the addition of irinotecan during HIPEC or 5-FU EPIC treatment resulted in improved DFS, both in the Kaplan-Meier analysis with log-rank test and in the multivariable Cox regression model with hazard ratios. The subgroup analysis with $\mathrm{CC} 1-3$ and liver metastases excluded confirmed this conclusion further with median DFS reaching 24 months. However, as with the preliminary results from the PRODIGE-7 study, an OS difference was not achieved (7). Nonetheless, this is probably explained by the fact that the patients will receive a wide array of future treatments. Further curative intent surgeries/interventions, different number of palliative lines of treatment including possibly new trial drugs, etc., make OS comparisons difficult to do without significantly increasing the sample size.

\section{The rationale for HIPEC}

PM from CRC is associated with a poor prognosis. For a long period of time these patients were considered palliative 
Table 3 Univariable and a priori multivariable Cox proportional analysis with DFS as endpoint

\begin{tabular}{|c|c|c|c|c|}
\hline Variable & \multicolumn{2}{|c|}{ Univariate analysis } & \multicolumn{2}{|c|}{ Multivariate analysis } \\
\hline Age (years) & $1.00(0.99-1.01)$ & 0.6 & $1.00(0.99-1.02)$ & 0.9 \\
\hline Gender male/female (n) & $1.16(0.85-1.60)$ & 0.4 & $0.86(0.55-1.35)$ & 0.8 \\
\hline Rectum vs. colon & $1.24(0.73-1.21)$ & 0.4 & $2.22(0.95-5.00)$ & 0.07 \\
\hline Node positive disease primary & $1.10(0.74-1.65)$ & 0.6 & $0.89(0.54-1.47)$ & 0.6 \\
\hline $\mathrm{PCl}$ & $1.05(1.03-1.07)$ & $<0.001$ & $1.03(1.01-1.06)$ & 0.005 \\
\hline Liver metastases & $1.32(0.79-2.19)$ & 0.3 & $2.29(1.25-4.19)$ & 0.008 \\
\hline Adjuvant chemotherapy given & $1.20(0.83-1.73)$ & 0.3 & $1.24(0.79-1.92)$ & 0.3 \\
\hline OX-HIPEC & Reference & & & \\
\hline OX-HIPEC + 5-FU EPIC & $0.76(0.44-1.31)$ & 0.3 & $0.48(0.25-0.92)$ & 0.03 \\
\hline OXIRI-HIPEC & $0.66(0.43-1.01)$ & 0.06 & $0.59(0.37-0.94)$ & 0.03 \\
\hline
\end{tabular}

DFS, disease-free survival; $\mathrm{Cl}$, peritoneal cancer index; CC, completeness of cytoreduction; HIPEC, hyperthermic intraperitoneal chemotherapy; OX-HIPEC, oxaliplatin-based HIPEC; 5-FU, 5-fluorouracil; EPIC, post-operative intraperitoneal chemotherapy; OXIRIHIPEC, oxaliplatin-irinotecan-based HIPEC.

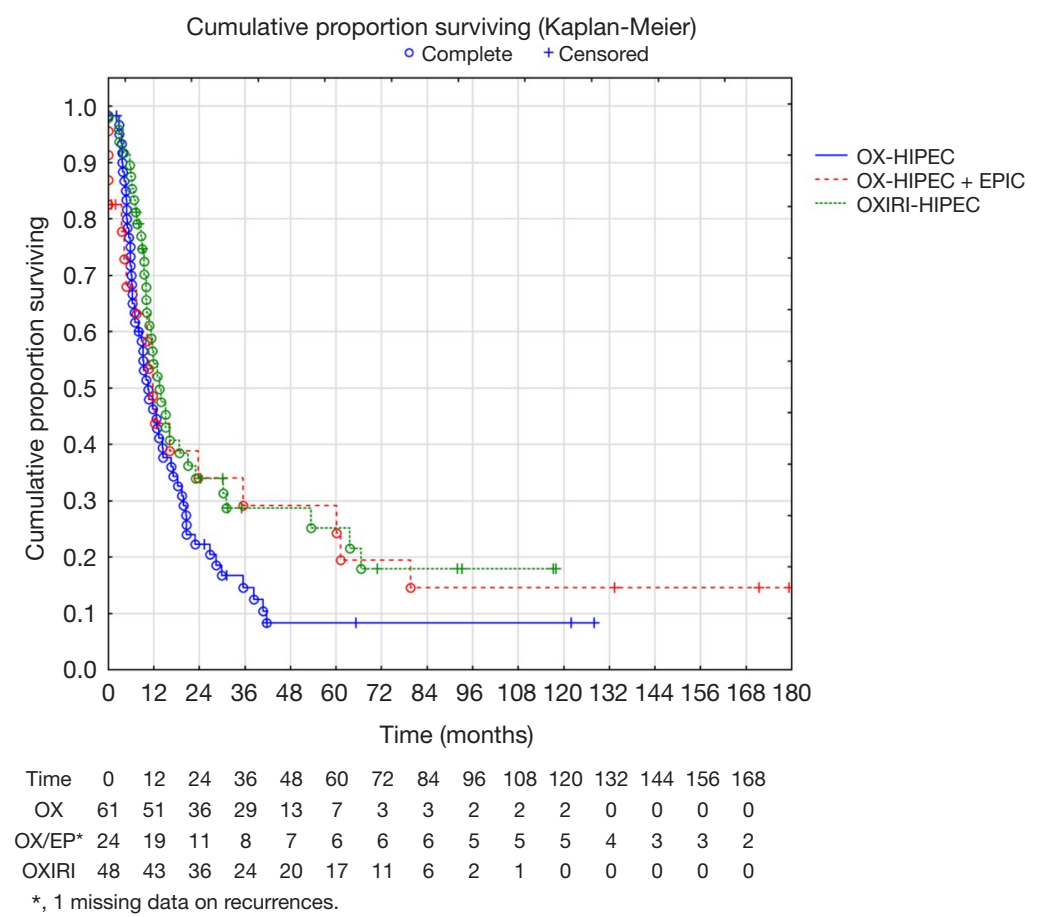

Figure 3 DFS between the three HIPEC regimens. OX-HIPEC vs. OX-HIPEC + EPIC P=0.33, OX-HIPEC vs. OXIRI-HIPEC P=0.049 (total $\mathrm{n}=133$ ). DFS, disease-free survival; HIPEC, hyperthermic intraperitoneal chemotherapy; OX-HIPEC, oxaliplatin-based HIPEC; EPIC, post-operative intraperitoneal chemotherapy; OXIRI-HIPEC, oxaliplatin-irinotecan-based HIPEC. 


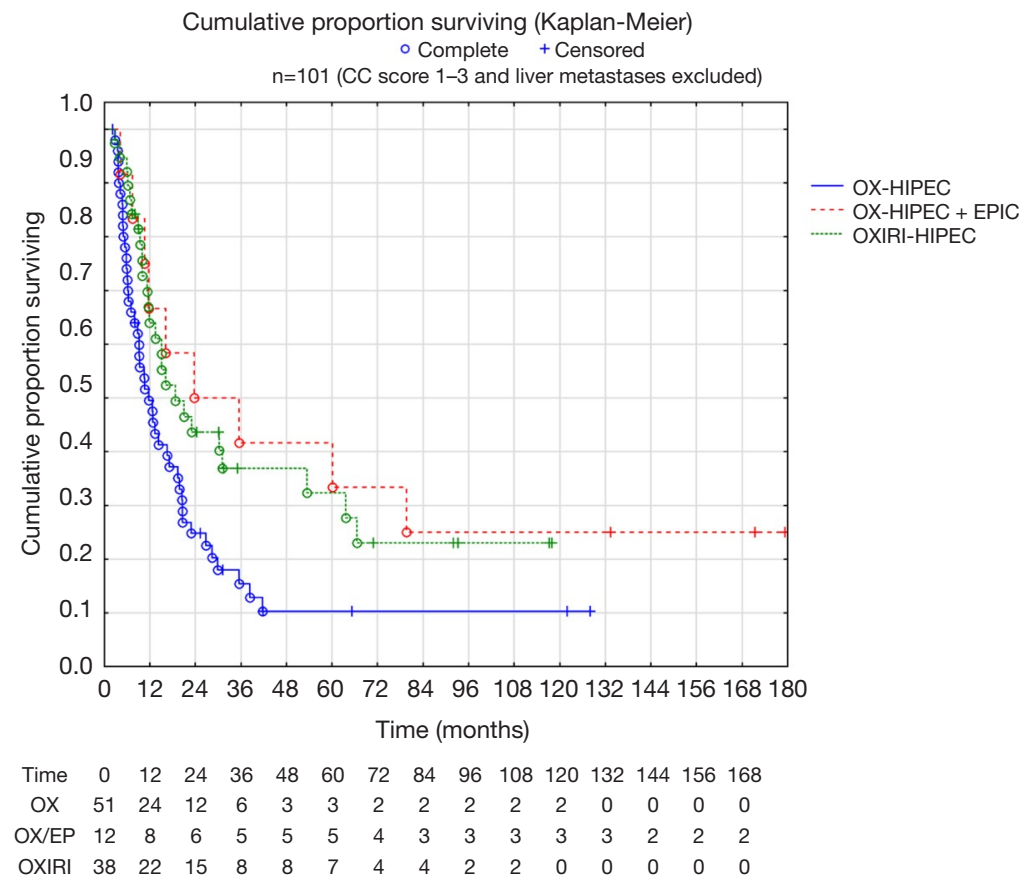

Figure 4 DFS between the three HIPEC regimens, excluding CC1-3 and liver metastases. OX-HIPEC $v s$. OX-HIPEC + EPIC P=0.029, OX-HIPEC $v s$. OXIRI-HIPEC $\mathrm{P}=0.017$ (total $\mathrm{n}=101$ ). DFS, disease-free survival; HIPEC, hyperthermic intraperitoneal chemotherapy; CC, completeness of cytoreduction; OX-HIPEC, oxaliplatin-based HIPEC; EPIC, post-operative intraperitoneal chemotherapy; OXIRIHIPEC, oxaliplatin-irinotecan-based HIPEC.

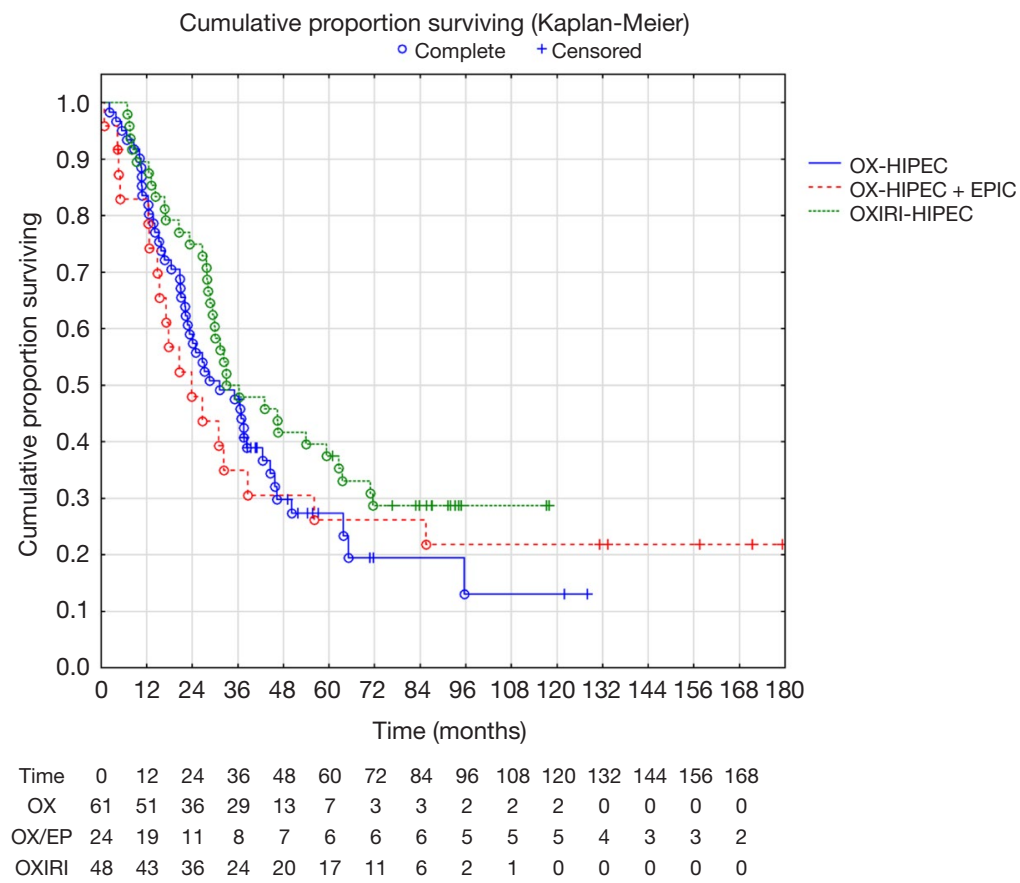

Figure 5 OS between the three HIPEC regimens. OX-HIPEC $v$ s. OX-HIPEC + EPIC P=0.9, OX-HIPEC vs. OXIRI-HIPEC P=0.16 (total $\mathrm{n}=133)$. OS, overall survival; HIPEC, hyperthermic intraperitoneal chemotherapy; OX-HIPEC, oxaliplatin-based HIPEC; EPIC, postoperative intraperitoneal chemotherapy; OXIRI-HIPEC, oxaliplatin-irinotecan-based HIPEC. 
with a median OS of 5-7 months (1). Advancements in chemotherapy, and molecular-targeted drugs, such as cetuximab and bevacizumab, may potentially extend median OS in these patients to nearly a year (14). Nevertheless, the prognosis is grim. In the last decades, CRS and HIPEC show promising results in patients in whom a complete macroscopic resection is achieved. Several studies have compared CRS and intraperitoneal chemotherapy, such as HIPEC and EPIC, with systemic chemotherapy in patients with CRC and PM (15-21).

A recent meta-analysis that included 76 studies, that assessed the therapeutic efficacy of CRS and HIPEC in patients with CRC and PM, concluded that HIPEC conferred significantly better survival in selected patients with CRC and PM (6). Unfortunately, the number of randomized control trials is limited, and there still remains a controversy regarding the role of HIPEC in patients with CRC and PM (17). Few previous studies have compared the long-term results of CRS and OX-HIPEC or OXHIPEC + EPIC and OXIRI-HIPEC in patients with CRC and $\mathrm{PM}(22)$.

\section{Baseline characteristics}

This retrospective study, that included 133 patients compared OX-HIPEC with OX-HIPEC + EPIC and OXIRI-HIPEC in patients with CRC and PM. Primary end-point was DFS, and secondary end-points morbidity and OS. All three treatment groups show similar baseline characteristics (Table 1). There are no differences with regards to age, gender, PCI, location of primary tumor, presence of liver metastases (although trend to more liver metastases in the intensified treatment groups, $\mathrm{P}=0.06$ ) or whether PM is metachronous or synchronous. Of note, is that there was a higher proportion of node-positive primary in the OX-HIPEC group compared to OXIRI-HIPEC. Furthermore, neo-adjuvant chemotherapy was more commonly given in the OXIRI-HIPEC group compared with the other HIPEC regimens. A CC score- 0 was similar across the three groups, and achieved in $89 \%$ of the cases in the whole cohort.

\section{DFS, OS and complications}

In contrast to Quenet et al. (22), DFS was better in patients that received intensified treatment with OXIRI-HIPEC compared to OX-HIPEC. DFS was 13.4 months compared to 10.5 months $(\mathrm{P}=0.049)$, and median OS was 35.6 months compared to 31.2 months $(\mathrm{P}=0.16)$. Five-year $\mathrm{OS}$ rate was $11 \%$ in OX-HIPEC, $25 \%$ in OX-HIPEC + EPIC, and $35 \%$ in OXIRI-HIPEC.

The multivariable Cox regression model showed that CC-score 1-3 vs. 0 was strongly associated with worse DFS (HR: 5.19, 95\% CI: 2.46-11.0). PCI and liver metastases were also identified in the multivariable analysis as associated with worse DFS. Additionally, intensification of HIPEC with either OX-HIPEC + EPIC or OXIRIHIPEC was associated with better DFS (Table 2). Morbidity according to Clavien-Dindo grades 3-4 were similar across the three treatment regimens. Neutropenia was significantly more common in the OXIRI-HIPEC group compared to OX-HIPEC and OX-HIPEC + EPIC $(\mathrm{P} \leq 0.0001)$. There were no differences in mortality between the three HIPEC treatments (Table 3).

A limitation of the present study is its retrospective non-randomized design. The proper impact of HIPEC on survival may therefore be difficult to assess due to selection biases. Even though these three regimens essentially represent three time periods, patient selection has been refined over the years. However, it is interesting to note that the most recent time period has been that of singleoxaliplatin use (the current national standard in Sweden) and OX-HIPEC with EPIC was used during the initial phase of the HIPEC program with the OXIRI HIPEC period in between. As such, it was unexpected that the multiple drug regimens would do so well in this comparison considering their early use.

Another potential limitation of this paper is the possibility of lead-time bias. That is, that the difference in DFS between the OXIRI-HIPEC and OX-HIPEC groups is merely a reflection of when and how often the scanning was performed. This is unlikely, however, given that all patients were followed-up similarly, according to national guidelines, with pre-specified time intervals between the imaging.

A common argument against HIPEC is that standard adjuvant systemic chemotherapy may have offered similar survival benefits for these highly selected patients. To answer, this question a randomized control trial is required. Nevertheless, this study provides important results concerning increased rates of DFS with intensified HIPEC treatments that can provide suggestions for future clinical trials. In Sweden, one such phase I/III trial program has recently been approved for funding by the Swedish Research Council. This program will dose-titrate a 1-day early EPIC with 5-FU and then move into a randomized 
trial where oxaliplatin HIPEC will be compared to oxaliplatin/irinotecan HIPEC + 1-day 5-FU EPIC for patients with CRC and PM disease (23).

\section{Conclusions}

An intensification of HIPEC by adding either irinotecan to oxaliplatin (OXIRI-HIPEC) or adding 5-FU EPIC resulted in better DFS compared to OX-HIPEC alone without an increased Clavien-Dindo morbidity or in-hospital mortality.

\section{Acknowledgments}

Funding: None.

\section{Footnote}

Reporting Checklist: The authors have completed the STROBE reporting checklist. Available at http://dx.doi. org/10.21037/jgo-20-494

Data Sharing Statement: Available at http://dx.doi. org/10.21037/jgo-20-494

Peer Review File: Available at http://dx.doi.org/10.21037/ jgo-20-494

Conflicts of Interest: All authors have completed the ICMJE uniform disclosure form (available at http://dx.doi. org/10.21037/jgo-20-494). The authors have no conflicts of interest to declare.

Ethical Statement: The authors are accountable for all aspects of the work in ensuring that questions related to the accuracy or integrity of any part of the work are appropriately investigated and resolved. The study was conducted in accordance with the Declaration of Helsinki (as revised in 2013), and was approved by the regional Ethical Review Board, DnR 2013/203. Because of the retrospective nature of the study, the Ethical Board did not consider a written informed consent to be necessary.

Open Access Statement: This is an Open Access article distributed in accordance with the Creative Commons Attribution-NonCommercial-NoDerivs 4.0 International License (CC BY-NC-ND 4.0), which permits the noncommercial replication and distribution of the article with the strict proviso that no changes or edits are made and the original work is properly cited (including links to both the formal publication through the relevant DOI and the license). See: https://creativecommons.org/licenses/by-nc-nd/4.0/.

\section{References}

1. Jayne DG, Fook S, Loi C, et al. Peritoneal carcinomatosis from colorectal cancer. Br J Surg 2002;89:1545-50.

2. Esquivel J, Elias D, Baratti D, et al. Consensus statement on the loco regional treatment of colorectal cancer with peritoneal dissemination. J Surg Oncol 2008;98:263-7.

3. Glehen O, Kwiatkowski F, Sugarbaker PH, et al. Cytoreductive surgery combined with perioperative intraperitoneal chemotherapy for the management of peritoneal carcinomatosis from colorectal cancer: a multiinstitutional study. J Clin Oncol 2004;22:3284-92.

4. Shen P, Hawksworth J, Lovato J, et al. Cytoreductive surgery and intraperitoneal hyperthermic chemotherapy with mitomycin $\mathrm{C}$ for peritoneal carcinomatosis from nonappendiceal colorectal carcinoma. Ann Surg Oncol 2004;11:178-86.

5. Elias D, Gilly F, Boutitie F, et al. Peritoneal colorectal carcinomatosis treated with surgery and perioperative intraperitoneal chemotherapy: retrospective analysis of 523 patients from a multicentric French study. J Clin Oncol 2010;28:63-8.

6. Huang CQ, Min Y, Wang SY, et al. Cytoreductive surgery plus hyperthermic intraperitoneal chemotherapy improves survival for peritoneal carcinomatosis from colorectal cancer: a systematic review and meta-analysis of current evidence. Oncotarget 2017;8:55657-83.

7. Quenet F, Elias D, Roca L, et al. A UNICANCER phase III trial of hyperthermic intra-peritoneal chemotherapy (HIPEC) for colorectal peritoneal carcinomatosis (PC): PRODIGE 7. J Clin Oncol 2018;36:abstr LBA3503.

8. von Elm E, Altman DG, Egger M, et al. The Strengthening the Reporting of Observational Studies in Epidemiology (STROBE) Statement: guidelines for reporting observational studies. Int J Surg 2014;12:1495-9.

9. Sugarbaker PH. Peritonectomy procedures. Ann Surg 1995;221:29-42.

10. Sugarbaker PH. Intraperitoneal chemotherapy and cytoreductive surgery for the prevention and treatment of peritoneal carcinomatosis and sarcomatosis. Semin Surg Oncol 1998;14:254-61.

11. Jacquet $\mathrm{P}$, Sugarbaker $\mathrm{PH}$. Clinical research methodologies in diagnosis and staging of patients with peritoneal carcinomatosis. Cancer Treat Res 
1996;82:359-74.

12. Sugarbaker PH. Management of peritoneal surface malignancy using intraperitoneal chemotherapy and cytoreductive surgery: a manual for physicians and nurses. 3rd ed. Grand Rapids: The Ludann Company, 1998.

13. Dindo D, Demartines N, Clavien PA. Classification of surgical complications: a new proposal with evaluation in a cohort of 6336 patients and results of a survey. Ann Surg 2004;240:205-13.

14. Adachi T, Hinoi T, Egi H, et al. Oxaliplatin and molecular-targeted drug therapies improved the overall survival in colorectal cancer patients with synchronous peritoneal carcinomatosis undergoing incomplete cytoreductive surgery. Surg Today 2015;45:986-92.

15. Elias D, Lefevre JH, Chevalier J, et al. Complete cytoreductive surgery plus intraperitoneal chemohyperthermia with oxaliplatin for peritoneal carcinomatosis of colorectal origin. J Clin Oncol 2009;27:681-5.

16. Franko J, Ibrahim Z, Gusani NJ, et al. Cytoreductive surgery and hyperthermic intraperitoneal chemoperfusion versus systemic chemotherapy alone for colorectal peritoneal carcinomatosis. Cancer 2010;116:3756-62.

17. Verwaal VJ, van Ruth S, de Bree E, et al. Randomized trial of cytoreduction and hyperthermic intraperitoneal chemotherapy versus systemic chemotherapy and palliative surgery in patients with peritoneal carcinomatosis of colorectal cancer. J Clin Oncol 2003;21:3737-43.

Cite this article as: Frühling P, Ghanipour L, Dranichnikov P, Enblad M, Birgisson H, Cashin PH. Oxaliplatin-based hyperthermic intraperitoneal chemotherapy with single drug versus multiple drug treatment for colorectal cancer with peritoneal metastases: an observational cohort study. J Gastrointest Oncol 2021;12(2):516-526. doi: 10.21037/jgo-20-494
18. Elias D, Delperro JR, Sideris L, et al. Treatment of peritoneal carcinomatosis from colorectal cancer: impact of complete cytoreductive surgery and difficulties in conducting randomized trials. Ann Surg Oncol 2004;11:518-21.

19. Glockzin G, Gerken M, Lang SA, et al. Oxaliplatin-based versus irinotecan-based hyperthermic intraperitoneal chemotherapy (HIPEC) in patients with peritoneal metastasis from appendiceal and colorectal cancer: a retrospective analysis. BMC Cancer 2014;14:807.

20. Cashin PH, Mahteme H, Syk I, et al. Quality of life and cost effectiveness in a randomized trial of patients with colorectal cancer and peritoneal metastases. Eur J Surg Oncol 2018;44:983-90.

21. Cashin PH, Mahteme H, Spang N, et al. Cytoreductive surgery and intraperitoneal chemotherapy versus systemic chemotherapy for colorectal peritoneal metastases: a randomised trial. Eur J Cancer 2016;53:155-62.

22. Quenet F, Goere D, Mehta SS, et al. Results of two biinstitutional prospective studies using intraperitoneal oxaliplatin with or without irinotecan during HIPEC after cytoreductive surgery for colorectal carcinomatosis. Ann Surg 2011;254:294-301.

23. Cashin PH, Sugarbaker PH. Hyperthermic intraperitoneal chemotherapy (HIPEC) for colorectal and appendiceal peritoneal metastases: lessons learned from PRODIGE 7. J Gastrointest Oncol 2020. doi: 10.21037/jgo2020-05. 